\title{
Two-stage bilateral laparoscopic adrenalectomy for large pheochromocytomas
}

\author{
Virgilijus Beisa, Michail Klimovskij, Gintaras Simutis, Audrius Sileikis, Kestutis Strupas \\ Faculty of Medicine, Vilnius University, Vilnius, Lithuania
}

Videosurgery Miniinv (e-pub, ahead of print) DOI: 10.5114/wiitm.2014.41005

\begin{abstract}
A 66-year-old Lithuanian female patient with a history of hypertension was diagnosed with bilateral adrenal tumors during a routine sonoscopy. Scintigraphy with metaiodobenzylguanidine and computed tomography scan revealed right 130/116/93 $\mathrm{mm}$ and left 85/61/53 $\mathrm{mm}$ pheochromocytomas. The patient suffered from hypertension with blood pressure over $240 / 100 \mathrm{~mm} \mathrm{Hg}$ and heartbeat disturbances. Blood adrenaline levels exceeded the norm 10-fold. After possible spread of tumors was rejected, laparoscopic transperitoneal adrenalectomy was planned in 2 stages, starting on the right then followed by the left side. After preoperative treatment with adrenoblockers, 2-stage bilateral laparoscopic adrenalectomy was performed. $13 \mathrm{~cm} \times 12 \mathrm{~cm} \times 9.5 \mathrm{~cm}$ right adrenal and, 3 months later, $8.5 \mathrm{~cm} \times 8 \mathrm{~cm} \times 6 \mathrm{~cm}$ left adrenal pheochromocytomas were removed. Histologically-radical extirpation, pheochromocytomas with possible malignant potential. Stable remission of hypertension was achieved postoperatively. Laparoscopic transperitoneal adrenalectomy is a safe and feasible method of treatment of large benign and possible malignant, but noninvasive pheochromocytomas.
\end{abstract}

Key words: pheochromocytoma, large pheochromocytoma, laparoscopic adrenalectomy, bilateral pheochromocytoma, laparoscopic transperitoneal adrenalectomy.

\section{Introduction}

Pheochromocytoma is a rare, catecholamine-secreting tumor arising mostly from the chromaffin cells of the adrenal gland, which clinically presents as a potentially curable form of hypertension. The annual incidence of pheochromocytomas is approximately $1-4 / 10^{6}$ population or $0.5 \%$ of subjects with hypertension [1]. In patients with inherited pheochromocytoma, benign and bilateral tumors are more common [2].

The diagnosis of pheochromocytoma is obtained by biochemical confirmation of catecholamine excess and imaging studies. Imaging studies used for diagnosis are ultrasound and computed tomography (CT) scan subsequently. Moreover, scintigraphy with me- taiodobenzylguanidine (MIBG), which is accumulated by adrenergic tissues, helps to determine an active pheochromocytoma diagnosis. Five-six percent of pheochromocytomas can be malignant [3]. The malignancy is confirmed by finding a metastatic spread; otherwise a benign diagnosis is established [4].

The only radical treatment of pheochromocytoma is adrenalectomy. Laparoscopic removal of pheochromocytoma is the preferred surgical technique, but, in case of malignancy, with signs of invasion, a transabdominal approach should be considered [5]. There were concerns about the size of tumor to be treated laparoscopically. However, developing minimally invasive techniques allows this method to be used for larger tumors [3]. The laparoscopic approach can be either transperitoneal or retroperitoneal. Substi-

\section{Address for correspondence}

Michail Klimovskij MD, Faculty of Medicine, Vilnius University, Perkunkiemio 19a-147, LT12120 Vilnius, Lithuania, phone: +37067110959,

e-mail: michail.klimovskij@gmail.com 
tutional therapy with corticosteroids postoperatively and long-term clinical follow-up of the patients are essential to obtain a good outcome [4].

We present a case of a patient with large bilateral pheochromocytomas, which were removed in two stages using the laparoscopic transabdominal technique.

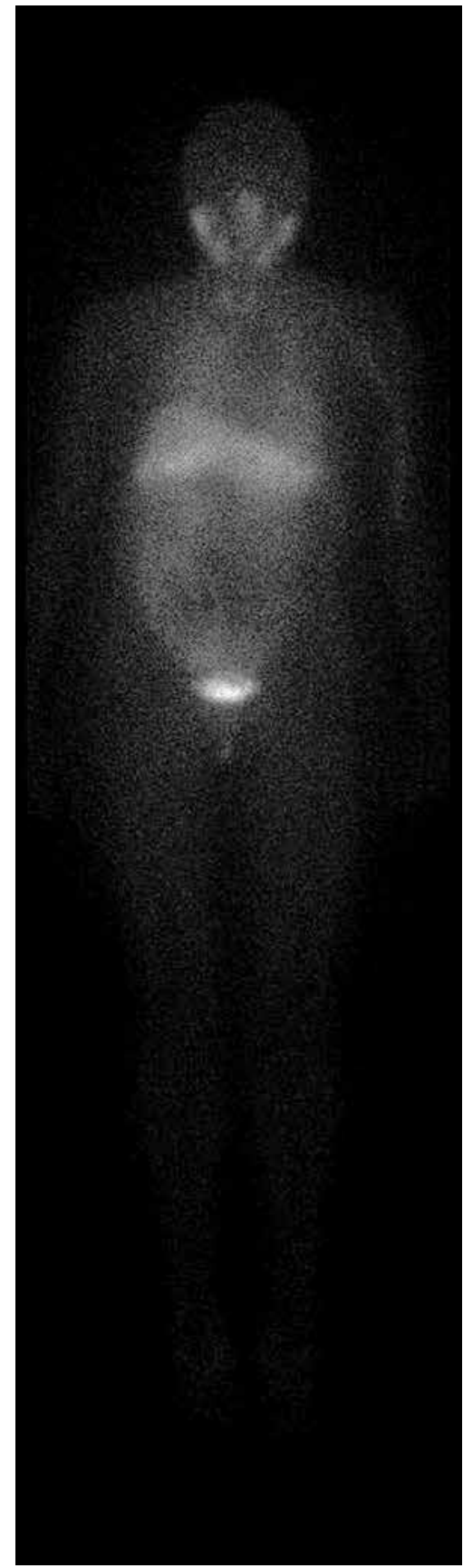

Photo 1. Body scintigraphy with late imaging (MIBG) revealed active pheochromocytomas

\section{Case report}

A 66-year-old Lithuanian female patient with a history of high blood pressure was incidentally diagnosed with bilateral adrenal tumors during a routine sonoscopy in December, 2009. Computed tomography (CT) scan showed a right $100 \mathrm{~mm} \times 76 \mathrm{~mm}$ adrenal tumor and $87 \mathrm{~mm} \times 58 \mathrm{~mm}$ left adrenal tumor with signs of cystic degeneration and no growth into surrounding structures. Blood adrenaline (1100 ng/l) levels exceeded the normal range 10-fold. Body scintigraphy with MIBG revealed right and left active pheochromocytomas (Photo 1). On the other hand, calcitonin levels were normal $(5.5 \mathrm{ng} / \mathrm{l})$, which eliminated the multiple endocrine neoplasia type 2A (MEN) syndrome diagnosis together with normal thyroid sonography results.

Operative treatment was suggested to the patient. However, the patient refused operation and further examination. Monitoring was resumed after the CT scan, performed in 2011, showed $10 \%$ growth of the right adrenal tumor $(110 \mathrm{~mm} \times 87 \mathrm{~mm})$. One year later the patient started to suffer from hypertension episodes with blood pressure over 240/100 mm Hg accompanied by headaches with vertigo and heartbeat disturbances. Computed tomography scan revealed right $130 / 116 / 93 \mathrm{~mm}$ and left 85/61/53 $\mathrm{mm}$ pheochromocytomas with clear boundaries, without invasion or growth into the surrounding structures. The right adrenal tumor was contacting and pressing the right liver lobe, inferior vena cava, right kidney and renal vein. The left adrenal tumor was contacting the pancreatic tail, posterior wall of the stomach, superior pole of the spleen, and also pressing and deforming the superior pole of the left kidney (Photo 1).

By that time the patient agreed to have an operation. Considering the fact that the tumors had not overgrown or invaded the surrounding organs in a 2-year period, the benign type of pheochromocytoma was confirmed, which provided the opportunity to select a laparoscopic approach for the resection of tumors. The laparoscopic adrenalectomy was planned in 2 stages, starting with the right and followed by left laparoscopic adrenalectomy 3 months later. A lateral transperitoneal approach was chosen for both operations. The patient received a 2-month course of the adrenoblockers phenoxybenzamine and metoprolol prior to the operation. 
Right laparoscopic adrenalectomy was started by placing the patient in a right lateral position. Four trocars were inserted $2 \mathrm{~cm}$ below the right costal arch as follows: midclavicular line $(10 \mathrm{~mm})$, anterior axillary line $(10 \mathrm{~mm})$, midaxillary line $(10 \mathrm{~mm})$, posterior axillary line $(5 \mathrm{~mm})$. After the right hepatic lobe was elevated with a retractor, the duodenum was mobilized using Kocher's approach. The $13 \mathrm{~cm}$ $\times 12 \mathrm{~cm} \times 9.5 \mathrm{~cm}$ adrenal gland was visualized between the inferior vena cava and superior renal pole. It was then separated using a harmonic scalpel and electrocoagulation. The $7 \mathrm{~mm}$ central adrenal vein was identified, stapled with an endo-GIA stapler and then cut. The separated gland was retrieved in a bag via the midclavicular port expanding the incision to $6 \mathrm{~cm}$ (Photos 3 and 4). Blood loss was $130 \mathrm{ml}$. Operation time was $210 \mathrm{~min}$. No severe blood pressure disturbances were observed during the adrenalectomy. The postoperative period passed without complications. The patient was discharged after 2 days. Histological findings proved the radical extirpation of pheochromocytoma, but with possible malignant potential: invasion to capsule, mitotic activity, focal necrosis.

Left laparoscopic adrenalectomy was performed 3 months later. The same position of trocars on the left side was established. The left colonic flexure and spleen were mobilized using a harmonic scalpel and electrocoagulation. The spleen was pushed with a retractor medially and the pancreatic tail was mobilized. The $8.5 \mathrm{~cm} \times 8 \mathrm{~cm} \times 6 \mathrm{~cm}$ left adrenal gland was found under the pancreatic tail medially from the left kidney's superior pole. The central vein was identified and clipped with a large clip in the site of outflow from the renal vein. The gland was separat-

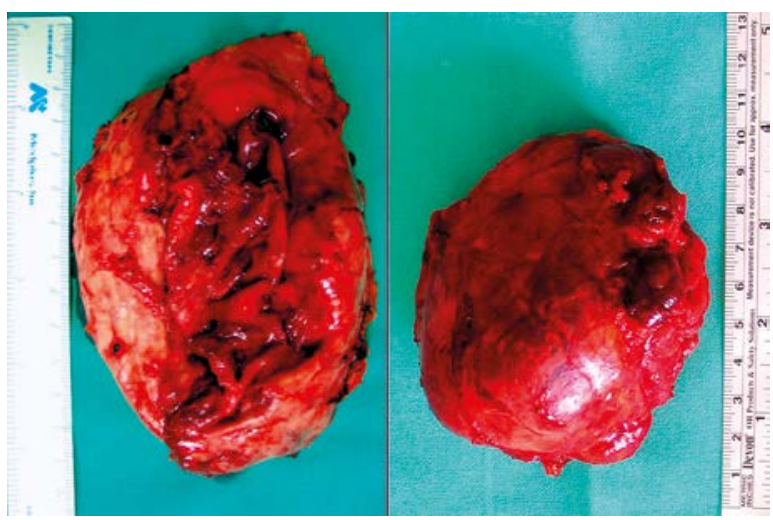

Photo 3. Right adrenal $(13 \mathrm{~cm})$ and left adrenal $(8.5 \mathrm{~cm})$ glands with pheochromocytomas

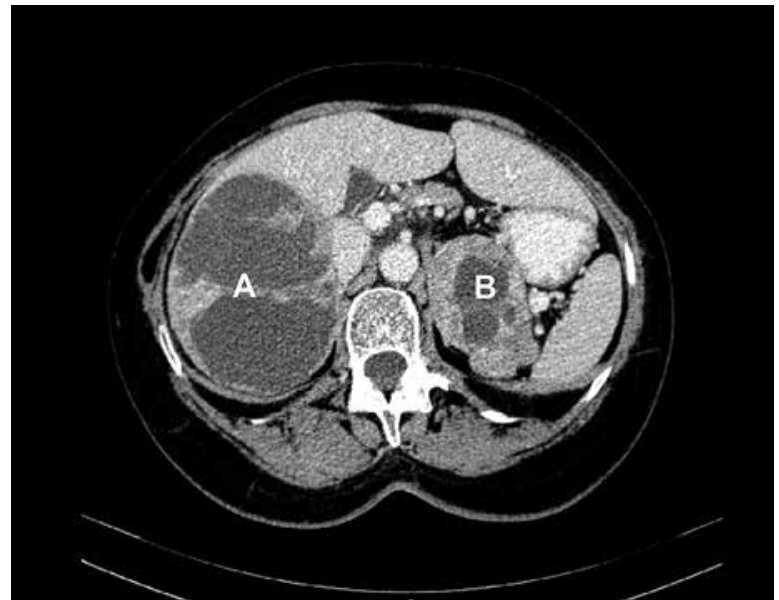

Photo 2. CT scan showed (A) right $130 \mathrm{~mm} \times$ $116 \mathrm{~mm} \times 93 \mathrm{~mm}$ and (B) left $85 \mathrm{~mm} \times 61 \mathrm{~mm}$ $\times 53 \mathrm{~mm}$ cystic adrenal tumors

ed and retrieved through the anterior axillary trocar site, expanding the incision to $5 \mathrm{~cm}$ length (Photos 3 and 4). Blood loss was $20 \mathrm{ml}$, operation time 180 min. Histological findings revealed similar radically resected pheochromocytoma with possible malignant potential. The postoperative course passed without complications. The patient was transferred to the endocrinological unit 2 days after surgery for adrenal hormone balance correction and was discharged on $45 \mathrm{mg}$ of hydrocortisone and $100 \mathrm{mg}$ fludrocortisone daily doses afterwards. Three months later chromogranin A level was 121.7 $\mathrm{ng} / \mathrm{ml}$ (normal < $148.56 \mathrm{ng} / \mathrm{ml}$ ). Scintigraphy with MIBG did not reveal any active accumulation spots 3 months postoperatively. Stable remission of hypertension and elimination of heartbeat disturbances were achieved.

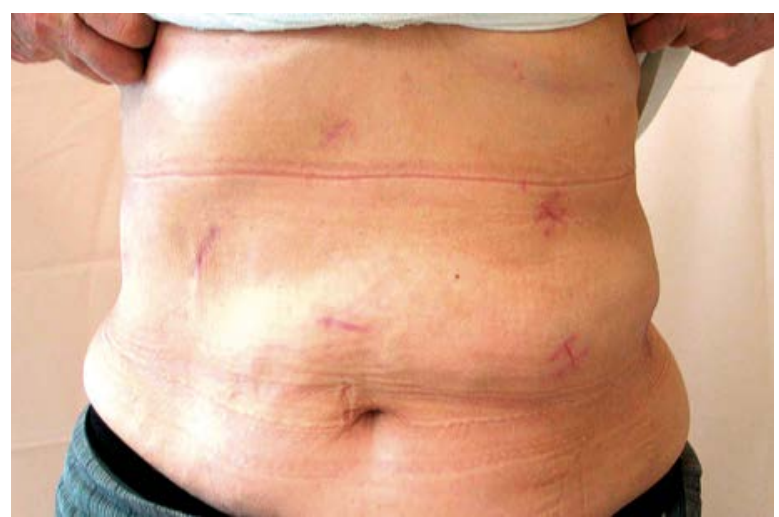

Photo 4. Scars of incisions 1 month after surgery 


\section{Discussion}

After laparoscopic surgery of the adrenal gland was first described in 1992 by Gagner, who used the transabdominal approach to access the gland in a patient with Cushing syndrome, this method has become a gold standard for managing benign adrenal lesions as well as pheochromocytomas [3, 5].

The initial goal for selection of laparoscopic treatment of pheochromocytoma is to determine whether a tumor is benign or malignant. Despite the concerns that large pheochromocytomas are more likely to be malignant, in our case, imaging studies showed no tumor overgrowth or spread into the surrounding structures and organs, which indicated a benign diagnosis.

A concern considering catecholaminergic discharge caused by the pneumoperitoneum did not seem sufficiently important to discard this technique as a first choice management. However, the procedure should be performed by an experienced surgeon, in order to reduce gland manipulation and shorten the operation time [6]. Early clipping and dividing of the adrenal vein helps to avoid a catecholamine-induced hypertensive crisis [3].

It is very important to control arterial blood pressure, heart rate and arrhythmias and to restore the blood volume to normal prior to surgery [7]. Preoperative treatment is usually started with administration of $\alpha$-adrenoblockers over a period of a fortnight. Additional $\beta$-adrenergic blockade can be required to treat any associated tachyarrhythmias. Our patient received an initial therapy with phenoxybenzamine $10 \mathrm{mg} 3$ times daily and metoprolol $25 \mathrm{mg}$ twice daily prior to the operation.

Which approach to choose? The retroperitoneal approach could be the primary choice in patients with severe cardiopulmonary disease in whom an increase in the $\mathrm{CO}_{2}$ level may occur, even in the presence of increased pulmonary ventilation [5]. On the other hand, the transperitoneal approach opens more workspace for manipulation. Also it may be beneficial for obese patients [8]. However, there is no significant difference between retroperitoneal and transperitoneal method outcomes, so the operating surgeon can select one of the mentioned approaches depending on his own experience and concerns $[5,8]$.

The retroperitoneal approach is feasible for tumors up to $6 \mathrm{~cm}$ in size. For tumors larger than $6 \mathrm{~cm}$ transabdominal laparoscopic technique should be preferred $[9,10]$. There is some evidence of suc- cessful laparoscopic adrenalectomy for an $18 \mathrm{~cm}$ tumor [9]. The main contraindication for laparoscopy is a malignant, $10 \mathrm{~cm}$ tumor $[9,11]$. Our case report demonstrates that laparoscopy could be used for tumors larger than $10 \mathrm{~cm}$ without signs of invasion to adjacent structures and organs.

The main reason for conversion is intraoperative bleeding as the result of splenic injury or tear of the vena cava during dissection of the right adrenal vein $[10,12]$. In our case major bleeding was avoided and the blood loss was minimal. Some sources suggest that left-sided adrenalectomy is an independent risk factor for overall and surgical complications after laparoscopic adrenalectomy [12]. It might be explained partly by the lateral transabdominal approach, which requires partial mobilization of the left pancreas and spleen. In our case bleeding during the left adrenalectomy was not significant.

Bilateral tumors may determine attempts to seek a genetic syndrome. Although the MEN syndrome diagnosis was eliminated after normal blood calcitonin and thyroid sonography results were obtained, a precise examination by a geneticist should be performed. Moreover, genetic evaluation can play an important role determining the malignancy and prognosis of pheochromocytoma [13]. Unfortunately, our patient refused genetic evaluation.

Histological findings revealed possible risk of malignancy. Pheochromocytoma of the adrenal gland scaled score (PASS) was 5 points for both sides (invasion to capsule 1 point; focal necrosis 2 points; mitotic activity 2 points). It is held that tumors with PASS below 4 have a benign behavior; on the other hand, those with PASS higher than 4 can act aggressively [14]. This is why a long-term follow-up of an oncologist is essential even if there were no signs of metastatic spread. Alternatively, normal blood chromogranin A level 3 months after the operation indicates a good short-term outcome [15].

\section{Conclusions}

Laparoscopic transperitoneal two-stage bilateral adrenalectomy is a safe and feasible method of treatment of large benign or malignant but noninvasive pheochromocytomas.

\section{References}

1. llias I, Pacak K. A clinical overview of pheochromocitomas/ paragangliomas and carcinoid tumors. Nucl Med Biol 2008; 35 (Suppl. 1): S27-34. 
2. Pederson LC, Lee JE. Pheochromocytoma. Curr Treat Options Oncol 2003; 4: 329-37.

3. Carter YM, Mazeh H, Sippel RS, Chen H. Laparoscopic resection is safe and feasible for large $(\geq 6 \mathrm{~cm})$ pheochromocytomas with out suspicion of malignancy. Endocr Pract 2012; 18: 720-6.

4. Parenti G, Zampetti B, Rapizzi E, et al. Updated and new perspectives on diagnosis, prognosis, and therapy of malignant pheochromocytoma/paraganglioma. J Oncol 2012; 2012: 872713.

5. Fernandez-Cruz L, Saenz A, Benarroch G, et al. Laparoscopic unilateral and bilateral adrenalectomy for Gushing's syndrome transperitoneal and retroperitoneal approaches Ann Surg 1996; 224; 727-36.

6. Aguilera Bazána A, Pérez Utrillaa M, Álvarez Escoláb C, et al. Laparoscopic adrenalectomy. Five-year experience [Spanish]. Actas Urol Esp 2010; 34: 181-5.

7. Ahmed A, Khan A. Perioperative management of pheochromocytoma: anaesthetic implications. JPMA 2007; 57: 140-6.

8. Nigri G, Rosman AS, Petrucciani N, et al. Meta-analysis of trials comparing laparoscopic transperitoneal and retroperitoneal adrenalectomy. Surgery 2013; 153: 111-9.

9. Fiszer P, Toutounchi S, Pogorzelski R, et al. Is tumour size a contraindication to laparoscopic adrenalectomy? Case report. Videosurgery Miniinv 2012; 7: 144-6.

10. Beisa V, Simutis G, Strupas K. Evaluation of two methods of endoscopic adrenalectomy. Zentralbl Chir 2009; 134: 1-5.

11. Stefanidis D, Goldfarb M, Kercher KW, et al. Guidelines for the minimally invasive treatment of adrenal pathology. Surg Endosc 2013; 27: 3960-80.

12. Gaujoux S, Bonnet S, Leconte M, et al. Risk factors for conversion and complications after unilateral laparoscopic adrenalectomy. Br J Surg 2011; 98: 1392-9.

13. Strong VE, Kennedy T, Al-Ahmadie $\mathrm{H}$, et al. Prognostic indicators of malignancy in adrenal pheochromocytomas: clinical, histopathologic, and cell cycle/apoptosis gene expression analysis. Surgery 2008; 143: 759-68.

14. Mlika M, Kourda N, Zorgati MM, et al. Prognostic value of Pheochromocytoma of the adrenal gland scaled score (Pass score) tests to separate benign from malignant neoplasms. Tunis Med 2013; 91: 209-15.

15. Szalat A, Fraenkel M, Doviner V, et al. Malignant pheochromocytoma: predictive factors of malignancy and clinical course in 16 patients at a single tertiary medical center. Endocrine 2011; 39 : 160-6.

Received: 31.07.2013, accepted: 5.11.2013. 